\title{
The clinical characteristics of posterior reversible encephalopathy syndrome in patients with chronic renal failure
}

\author{
HONGTAO HU ${ }^{1}$, SHEN XU ${ }^{1}$, SHUANG HU ${ }^{1}$, WEIJIA XU $\mathrm{XU}^{1,2}$ and HUA SHUI ${ }^{1}$ \\ ${ }^{1}$ Department of Nephrology, Zhongnan Hospital of Wuhan University, Wuhan, Hubei 430071; \\ ${ }^{2}$ Department of Nephrology, Taihe Hospital, Shiyan, Hubei 442000, P.R. China
}

Received April 27, 2016; Accepted March 23, 2017

DOI: $10.3892 /$ etm.2017.4570

\begin{abstract}
Few studies have investigated posterior reversible encephalopathy syndrome (PRES) in patients with chronic renal failure (CRF). The present study analyzed the clinical manifestations, laboratory examinations and imaging features of PRES in patients with CRF. A total of 42 patients with CRF with or without PRES were recruited in the current retrospective case-control study. Patient data taken prior to the onset of PRES in patients with CRF and PRES $(n=21)$ were collected and analyzed. At the same time, data from patients with CRF but without PRES $(\mathrm{n}=21)$ were also analyzed. Brain magnetic resonance imaging (MRI) scans were collected from patients in the PRES group. The mean blood pressure of patients in the PRES group was significantly higher than that of the control group (systolic blood pressure: $172 \pm 15 \mathrm{mmHg}$ vs. $135 \pm 14 \mathrm{mmHg}, \mathrm{P}<0.01$; diastolic blood pressure: $95 \pm 16 \mathrm{mmHg}$ vs. $64 \pm 13 \mathrm{mmHg}, \mathrm{P}<0.01)$. Furthermore, compared with the control group, mean serum albumin (Alb) and hemoglobin ( $\mathrm{Hb}$ ) concentrations in the PRES group were significantly lower (Alb: $29.1 \pm 5.3 \mathrm{~g} / \mathrm{l}$ vs. $34.6 \pm 6.1 \mathrm{~g} / \mathrm{l}, \mathrm{P}=0.001 ; \mathrm{Hb}: 74 \pm 16 \mathrm{~g} / \mathrm{l}$ vs. $89 \pm 28 \mathrm{~g} / 1, \mathrm{P}=0.037)$. By contrast, mean LDH concentration was significantly higher in the PRES group (LDH: $336 \pm 141 \mathrm{U} / 1$ vs. $235 \pm 89 \mathrm{U} / \mathrm{l}, \mathrm{P}=0.004)$. In the PRES group, $24 \mathrm{~h}$ urine volume was significantly lower in the PRES group than in the control group ( $24 \mathrm{~h}$ urine volume: $651 \pm 520 \mathrm{ml}$ vs. $982 \pm 518 \mathrm{ml}$, $\mathrm{P}=0.046$ ). No significant differences in levels of serum potassium $(4.5 \pm 0.6 \mathrm{mmol} / \mathrm{l}$ vs. $4.4 \pm 0.5 \mathrm{mmol} / \mathrm{l}, \mathrm{P}=0.377)$, sodium (138.3 $\pm 4.9 \mathrm{mmol} / \mathrm{l}$ vs. $139.0 \pm 6.8 \mathrm{mmol} / \mathrm{l}, \mathrm{P}=0.325)$, calcium $(2.0 \pm 0.24 \mathrm{mmol} / 1$ vs. $1.9 \pm 0.24 \mathrm{mmol} / \mathrm{l}, \mathrm{P}=0.673)$, alanine aminotransferase; $(24 \pm 14 \mathrm{U} / \mathrm{l}$ vs. $18 \pm 8 \mathrm{U} / 1, \mathrm{P}=0.975)$; aspartate aminotransferase $(29 \pm 11 \mathrm{U} / 1$ vs. $24 \pm 9 \mathrm{U} / 1, \mathrm{P}=0.619)$ and uric acid $(448 \pm 148 \mu \mathrm{mol} / 1 \mathrm{vs.} 378 \pm 116 \mu \mathrm{mol} / 1, \mathrm{P}=0.599)$ were found
\end{abstract}

Correspondence to: Dr Hua Shui, Department of Nephrology, Zhongnan Hospital of Wuhan University, 169 Road Donghu, Wuhan, Hubei 430071, P.R. China

E-mail: shuihua2005@aliyun.com

Key words: chronic renal failure, posterior reversible encephalopathy syndrome, imageology, biochemical tests, clinical manifestations between the two groups. PRES is a relatively common nervous system complication arising in patients with CRF. Certain biochemical markers, including $\mathrm{Hb}$ and $\mathrm{Alb}$, may be associated with PRES. Diagnosing PRES is difficult as computed tomography (CT) brain scans may be normal and MRI scans, which are more sensitive than CT scans at diagnosing PRES, are not always performed in patients with CRF. Thus, brain MRI scans should be taken first in such patients when PRES is suspected.

\section{Introduction}

Chronic renal failure (CRF) is a clinical syndrome of severe renal impairment caused by a number of predisposing factors, such as diabetes, hypertension, hyperuricaemia and glomerulonephritis (1). Neurological complications, such as posterior reversible encephalopathy syndrome (PRES), are relatively common in patients with CRF and $\sim 45 \%$ of the reported kidney disease cases exhibit comorbidity with PRES (2).

PRES is a well-known reversible neuroradiological clinical entity characterized by symptoms including headaches, nausea and vomiting, seizures, alterations in consciousness, vision changes, cortical blindness and other focal neurologic symptoms and signs (2). The incidence of PRES is moderately higher in females than in males (male:female ratio is $0.8: 1$ ) and the mean age at presentation is 44 years with an extended age range of 14 to 78 years (2). PRES is typically diagnosed based on clinical symptom, imaging examination (computed tomography or magnetic resonance images) and predisposing factors such as acute hypertension, preeclampsia, cytotoxic or immunosuppressive medications (particularly cyclosporine) and connective tissue diseases (3). In addition, the most characteristic abnormality of PRES identified by neuroimaging is the presence of posterior white matter edema with symmetrical involvement of the parietal and occipital lobes for vascular cerebral dysregulation (4). The symptoms of PRES are generally reversible, however, in certain cases, cerebral hemorrhage or ischemia may occur and result in irreversible neurological deficits or death (2). The etiology of PRES is not fully understood and there are a number of hypotheses regarding its mechanism of action (5). Previous studies have demonstrated that PRES is associated with different clinical syndromes (6-10) including acute hypertension, preeclampsia/eclampsia, antineutrophil cytoplasmic autoantibody-associated vasculitis, 
systemic lupus erythematosus and renal insufficiency. The majority of studies concerning PRES have only investigated its neurological symptoms and imaging findings and have not performed statistical analyses of biochemical indicators relevant to PRES $(4,11)$. Renal dysfunction is a known cause of PRES (2), however the majority of patients with renal failure do not develop PRES, meaning that the exact nature of the relationship between CRF and PRES is unknown. To the best of our knowledge, there have been no studies investigating the potential relationship between CRF and PRES.

The present study summarized the biochemical indicators and clinical characteristics of PRES patients with CRF, as well as associated factors that may be involved in the development of PRES, in order to determine the potential pathophysiological mechanism of PRES in patients with CRF and improve the diagnosis and treatment of PRES.

\section{Patients and methods}

Data collection. All patients recruited in the present study were required to satisfy the following conditions: i) The diagnosis of CRF was confirmed in our hospital (Zhongnan Hospital of Wuhan University, Wuhan, China) and the course of disease had lasted for $\geq 3$ months; ii) the guidelines of the American Kidney Disease Foundation K/DOQI (12) were followed: Glomerular filtration rate (GFR) was used as the classification criteria and only patients between chronic kidney disease (CKD) 3 and CKD5 (based on the five-stage CKD classification where CKD1, CKD2, CKD3, CKD4 and CKD5 have an estimated GFR range of $>90,60-89$, 30-59, 15-29 and $<15 \mathrm{ml} / \mathrm{min} / 1.73 \mathrm{~m}^{2}$, respectively) (12) were selected; and iii) patients with additional conditions, including severe lung infection, acute blood loss, acute gouty arthritis, acute coronary syndrome and multiple organ dysfunction syndrome, were excluded from the current study.

A total of 42 patients were recruited in the current study from the Zhongnan Hospital of Wuhan University (Hubei, China) between June 2009 and June 2014. A total of 21 patients with both PRES and CRF were included in the PRES group and a further 21 patients with CRF but without PRES were included as a control group. Diagnoses of PRES were made using the following criteria (13): i) Acute or subacute clinical symptoms including headache, altered mental status, seizures or visual disturbances with or without elevated systemic blood pressure; ii) the presence of known risk factors including hypertension, administration of immunosuppressive drugs and severe electrolyte disturbances; iii) abnormalities identified by neuroimaging, including distributions of brain lesions identified by magnetic resonance imaging (MRI) or computed tomography (CT) that are compatible with a typical PRES lesion pattern, as well as MRI brains exhibiting high signal intensity on T2-weighted images (T2WI) and fluid-attenuated inversion recovery images (FLAIR); iv) Rapid recovery from PRES-related illness, including the resolution of clinical abnormalities following proper therapy, for example, treatment of seizures with anti-epileptic drugs, treatment of hypertension with anti-hypertensive agents and discontinuation of this medication, at least temporarily, in the acute setting if PRES is caused by a specific medication (such as antirejection drugs); v) the ruling out of other confusable encephalopathy syndromes, including metabolic encephalopathy, autosomal dominant leukoencephalopathy, reversible cerebral vasoconstriction syndrome and antibiotic encephalopathy.

Neuroimaging reviews were performed by two independent and certified radiologists blinded to the clinical features of the patients, who were trained in diagnosing PRES via MRI. The location, distribution, severity of any signal abnormalities and presence of hemorrhage were appraised and itemized. The results of the neuroimaging reviews from the two radiologists were consistent and any conflicts between the assessments of the reviewers were resolved by consensus. The protocol of the current study was approved by the Institutional Ethics Review Boards of Zhongnan Hospital (Wuhan, China). Informed consent from patients was waived as this was a retrospective study. The study protocol conformed to the ethical guidelines of the Declaration of Helsinki and all patient information was anonymized during data collected and analysis.

Research methods. As part of the diagnostic evaluation, all patients in the PRES group underwent MRI or CT within several $h$ to one day after the onset of symptoms as described above. The choice of which manner to diagnose was based on the decision of the patients and their family, even though we recommended MRI to patients and their family. MRI studies were performed at 1.5T MRI scanners (Siemens Avanto 1.5T, Siemens AG, Munich, Germany). Imaging sequences included T1WI, T2WI and FLAIR. CT studies were performed on a multi-slice spiral CT (Siemens Sensation 16; Siemens AG).

Statistical analysis. All data were analyzed using SPSS 13.0 (SPSS, Chicago, IL, USA) statistical software. Measurement data were expressed as the mean \pm standard deviation and counting data were expressed as case number and percentages. Measurement data were assessed using the Student's t-test, counting data were assessed using the $\chi^{2}$ test. Intergroup comparisons were performed using Mann-Whitney U test for hierarchical variables of two independent samples. $\mathrm{P}<0.05$ was considered to indicate a significant difference.

\section{Results}

General conditions. Table I presents the general conditions, including the sex ratio, mean age, dialysis ratio and constituent ratio in different stages. A total of 21 patients ( 7 males and 14 females) with PRES were included in the current study. Mean patient age was $65.4 \pm 9.5$ years (range, $41-79$ years) and 16 patients in the PRES group were under dialysis $(n=13$ in the control group). The numbers of patients in the CKD3/CKD4/CKD5 stages were $2 / 2 / 17$ respectively. There were no statistical differences in the sex ratio, mean age, dialysis ratio $(\mathrm{P}>0.05)$ and constituent ratio $(\alpha=0.05, \mathrm{P}>0.05)$ between the two groups, highlighting that there were no significant differences in the baseline characteristics of the two groups.

Blood pressure (BP). During the 48-h data collection, all BP indicators including average systolic blood pressure (SBP), diastolic blood pressure (DBP) and mean arterial pressure (MAP) were significantly higher in patients with PRES compared with the control group $(\mathrm{P}<0.05$; Fig. 1). 
Table I. Patient clinical features.

\begin{tabular}{lcccc}
\hline Group & Male/female & Mean age (years) & Dialysis/total & CKD3/CKD4/CKD5 \\
\hline Control & $11 / 10$ & $63.4 \pm 10.5$ & $13 / 21$ & $3 / 5 / 13$ \\
PRES & $7 / 14$ & $65.4 \pm 9.5$ & $16 / 21$ & $2 / 2 / 17$ \\
P-value & 0.212 & 0.518 & 0.317 & 0.205 \\
\hline
\end{tabular}

$\mathrm{n}=21$. PRES, posterior reversible encephalopathy syndrome; CKD, chronic kidney disease.

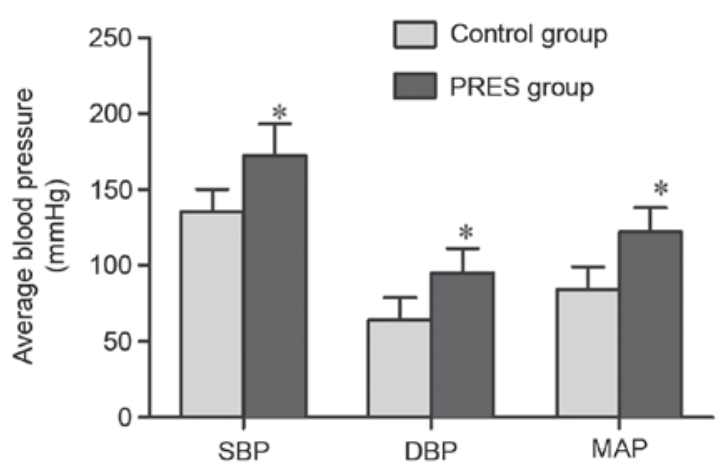

Figure 1. Average blood pressure measurements in patients with posterior reversible encephalopathy syndrome and control groups over 48 h. ${ }^{*} \mathrm{P}<0.05$ vs. control group. DBP, diastolic blood pressure; SBP, systolic blood pressure; MAP, mean arterial pressure.

Neurological symptoms. At the time of the neurological event indicated by abnormal CT or MRI results, patients with PRES presented with headache $(85.6 \%)$, seizures $(80.9 \%)$, nausea/vomiting $(47.6 \%)$, visual disturbance $(38.1 \%)$ or consciousness disorder (19.0\%; Fig. 2). The proportion of patients presenting with such symptoms differed from that reported by Yamada et al (14). Patients in the control group presented with none of the aforementioned neurological symptoms.

Biochemical results. Biochemical results are summarized in Table II. Compared with the control group, the average hemoglobin $(\mathrm{Hb})$ and serum albumin (Alb) levels and total urine volume over $24 \mathrm{~h}$ were all significantly lower in the PRES group compared with the control $(\mathrm{P}<0.05)$. By contrast, serum lactate dehydrogenase levels $(\mathrm{LDH})$ were significantly greater in the PRES group compared with the control $(\mathrm{P}<0.05$; Table II).

Neuroimaging findings. In the PRES group, $76 \%$ of patients (16/21) underwent CT head scans as 5 patients did not wish to undergo MRI. Of these 16 patients, 13 exhibited features of PRES following the scan. Thus, the sensitivity of CT in diagnosing PRES was $81.3 \%$ (13/16). Abnormal CT images exhibited atypical hypodensities, which were symmetrically located in the white matter of rear hemisphere (Fig. 3A-C).

At the same time, 17 (17/21) patients in the PRES group underwent a brain MRI scan. All of patients were accurately diagnosed as having PRES, demonstrating the sensitivity of MRI to PRES was $100 \%$. The lesions were obvious on MRI, which exhibited high signal intensity in bilateral frontal lobe, parietal lobe, occipital lobe, posterior temporal lobe, parietal

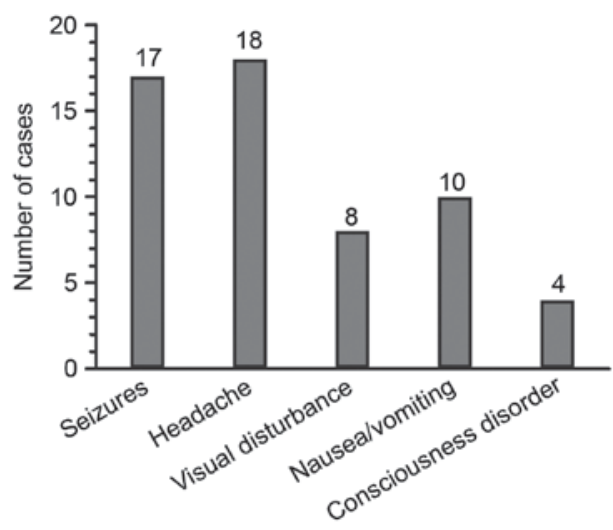

Figure 2. Number of patients with different neurological symptoms in the posterior reversible encephalopathy syndrome group.

cortex and subcortical areas in bilateral cerebellum on brain T2/fluid-attenuated inversion recovery (FLAIR) MRI and low signal intensity on T1WI (Fig. 3D-F). Furthermore, smaller lesions on CT were easily missed unless they were quite clear or on typical lesion sites. Therefore, MRI was more advantageous than CT at identifying slight lesions (Fig. 4). Taken together, the results of the current study indicate that MRI is more sensitive than CT scans at diagnosing PRES (detection rate: $100 \%$ vs. $81.3 \%$ ).

\section{Discussion}

PRES is a clinical and radiological syndrome characterized by reversible vasogenic edema in the posterior brain, which primarily arises from autoregulation failure and endothelial dysfunction (2). There are a number of causes that may lead to the development of PRES. Malignant hypertension, pre-eclampsia/eclampsia, immune-related diseases (14-16) and tumor-related diseases have been reported as potential causes of PRES $(17,18)$. previous studies have indicated that patients with CRF are more likely to develop PRES in certain cases $(19,20)$.

The pathogenesis of PRES has not yet been determined. Currently, there are three main hypotheses $(7,15,21)$ : i) The cerebral hyperperfusion theory, stating that blood pressure rises so highly and rapidly that it goes exceeds the upper limit of cerebrovascular autoregulation. This dysfunction stimulates small arteries to undergo expansion rather than contraction and leads to cerebral hyperperfusion. Following this, water molecules and macromolecules undergo exosmosis to the brain cell mesenchyme through the damaged 
Table II. Patient biochemical results.

\begin{tabular}{|c|c|c|c|c|}
\hline Group & Control & PRES & T-value & P-value \\
\hline $\mathrm{Hb}(\mathrm{g} / \mathrm{l})$ & $89 \pm 28$ & $74 \pm 16$ & -2.252 & $0.037^{\mathrm{a}}$ \\
\hline $\mathrm{Alb}(\mathrm{g} / \mathrm{l})$ & $34.6 \pm 6.1$ & $29.1 \pm 5.3$ & -3.426 & $0.001^{\mathrm{a}}$ \\
\hline $\mathrm{K}(\mathrm{mmol} / \mathrm{l})$ & $4.4 \pm 0.5$ & $4.5 \pm 0.6$ & 0.905 & 0.377 \\
\hline $\mathrm{Na}(\mathrm{mmol} / \mathrm{l})$ & $139.0 \pm 6.8$ & $138.3 \pm 4.9$ & 1.011 & 0.325 \\
\hline $\mathrm{Ca}(\mathrm{mmol} / \mathrm{l})$ & $1.9 \pm 0.24$ & $2.0 \pm 0.24$ & -0.429 & 0.673 \\
\hline $\mathrm{P}(\mathrm{mmol} / \mathrm{l})$ & $1.3 \pm 0.47$ & $1.7 \pm 0.59$ & 1.587 & 0.128 \\
\hline $\operatorname{ALT}(\mathrm{U} / \mathrm{l})$ & $18 \pm 8$ & $24 \pm 14$ & -0.032 & 0.975 \\
\hline AST (U/l) & $24 \pm 9$ & $29 \pm 11$ & 0.506 & 0.619 \\
\hline $\mathrm{UA}(\mu \mathrm{mol} / \mathrm{l})$ & $378 \pm 116$ & $448 \pm 148$ & 0.531 & 0.599 \\
\hline $\operatorname{Scr}(\mu \mathrm{mol} / 1)$ & $488 \pm 255$ & $532 \pm 243$ & -0.574 & 0.937 \\
\hline $\mathrm{LDH}(\mathrm{U} / \mathrm{l})$ & $235 \pm 89$ & $366 \pm 141$ & 3.335 & $0.004^{\mathrm{a}}$ \\
\hline Total urine volume over $24 \mathrm{~h}(\mathrm{ml})$ & $982 \pm 518$ & $651 \pm 520$ & -2.064 & $0.046^{\mathrm{a}}$ \\
\hline
\end{tabular}

Data are presented as the mean \pm standard deviation. $\mathrm{n}=21 .{ }^{\mathrm{a}} \mathrm{P}<0.05$ between the two groups. Hb, hemoglobin; Alb, serum albumin; ALT, alanine aminotransferase; AST, aspartate aminotransferase; UA, uric acid; Scr, serum creatinine; LDH, serum lactate dehydrogenase.

blood-brain barrier, resulting in the onset of cerebral edema. ii) The vasospasm theory, stating that cerebrovascular serves the important function of maintaining cerebral perfusion at a stable level. When blood pressure rises sharply, excessive regulation of the cerebrovascular system at that time will induce cerebrovascular vasospasm. This reduces blood flow into the brain capillaries, resulting in the onset of cerebral ischemia. Cerebrovascular hypoperfusion and ischemia reperfusion both injure the vascular endothelium, resulting in acute endothelial dysfunction. Due to this, the vascular permeability of the brain increases and intravascular proteins and fluid penetrate into the brain mesenchyme, causing cerebral edema. iii) The damaged vascular endothelium theory, which is completely different from aforementioned two theories. Certain scholars consider that the primary mechanism of PRES is dysfunction of the vascular endothelium. For patients with CRF, uremic toxins, inflammatory cytokines and oxidative stress may all lead to endothelial dysfunction $(22,23)$. Furthermore, immunosuppressive agents and cytotoxic drugs are frequently used to treat PRES and may also directly cause endothelial dysfunction (24,25). Even hemodialysis itself may be associated with the occurrence and aggravation of PRES, as it disrupts the state of equalization of the cerebrovascular between the exterior and interior and may lead to endothelial damage through different pathways, such as loss of nitric oxide signaling (26,27). Piccin et al (17) analyzed two cases of acute lymphoblastic leukemia that developed into PRES during induction chemotherapy to decrease anti-thrombin-III levels. This may provide a novel insight into PRES pathophysiology that a reduction in anti-thrombin-III may cause an increase in endothelial cell permeability and stimulate the leakage of plasma, Alb and red blood cells into the extracellular space, thus inducing PRES. For patients with CRF, endothelial injury and dysfunction seems to be the common pathway and primary mechanism of PRES development.

In the present study, all measures of BP in the PRES group were significantly higher than those of the control group which indicated that the BP parameters, including SBP, DBP and MAP may be correlated with the onset of PRES. Hypertension is an important pathophysiological mechanism that occurs during the development of PRES. It is not only a primary clinical feature of PRES but also an important factor contributing to the collapse of cerebrovascular autoregulation (28). However, in the current study, there were 5 patients with normal blood pressure in the PRES group, indicating that not all patients with PRES were hypertensive.

Neuroimaging findings are essential to the diagnosis of PRES. CT images of the brain exhibited atypical hypodensities, the shape of which were irregular. Compared with head CT, brain MRI images were more characteristic: They revealed lesions as low or equivalent signal intensity on T1WI and high signal intensity on T2WI and FLAIR. Minimal lesions were easily missed by the CT scan unless they were found at typical lesion sites. In the current study, MRI was more sensitive than CT in the diagnosis of PRES (detection rate of $100 \%$ vs. $81.3 \%$ ). Therefore, due to the fact that a CT brain scan of patients with PRES may be normal and MRI scans are more accurate in diagnosing PRES, a brain MRI rather than a CT scan should be performed in all cases when PRES is highly suspected.

In the current study, average levels of serum Alb in the PRES group were significantly lower than those of the control group $(\mathrm{P}<0.05)$. This suggests that low serum Alb levels may be an important contributing factor to PRES in patients with CRF. It is thus worth investigating further the role hypoproteinemia serves in PRES. One possibility is that hypoalbuminemia may contribute to progressive damage to vascular endothelial cells, leading to endothelial dysfunction (29). Due to this dysfunction, serum Alb may leak out of the vascular endothelium, resulting in a reduction of plasma colloid osmotic pressure, which leads to fluid exudation and vascular cerebral edema (29). Another possible mechanism is that endothelial dysfunction increases vascular permeability, causing constant Alb leakage and hypoproteinemia, consequently resulting in the development of cerebral edema (30). The exact mechanism 

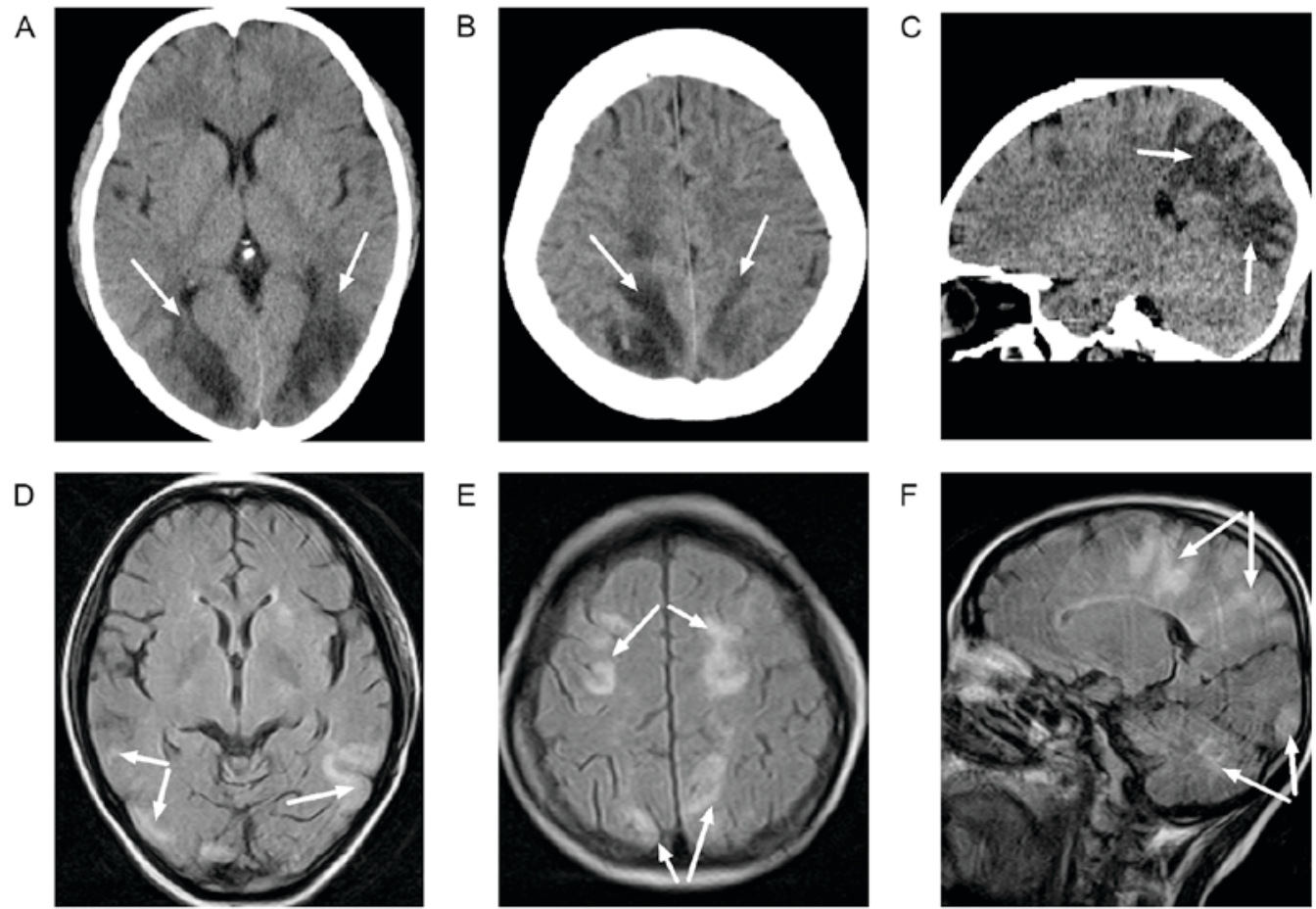

Figure 3. (A, B and C) PRES lesions following head CT. Abnormal CT images exhibited atypical hypodensities. (A) Extensive low density shadow on the bilateral occipital lobe. (B) Extensive low density shadow on the bilateral parietal lobe. (C) Sagittal reconstruction indicated multiple low density shadow on the occipital lobe. (D, E and F) PRES lesions exhibited high signal intensity on brain T2 FLAIR MRI. (D) T2 MRI-FLAIR indicated multiple high signal lesions in bilateral posterior temporal lobe and right occipital cortex. (E) T2 MRI-FLAIR revealed multiple high signal lesions in bilateral frontal, parietal cortex and subcortical areas. (F) T2 sagittal MRI-FLAIR showed high signal lesions in bilateral cerebellum, frontal lobe, parietal lobe and occipital lobe, as indicated by the arrows in the figures. PRES, posterior reversible encephalopathy syndrome; MRI, magnetic resonance imaging; FLAIR, fluid-attenuated inversion recovery images; $\mathrm{CT}$, computed tomography.

A

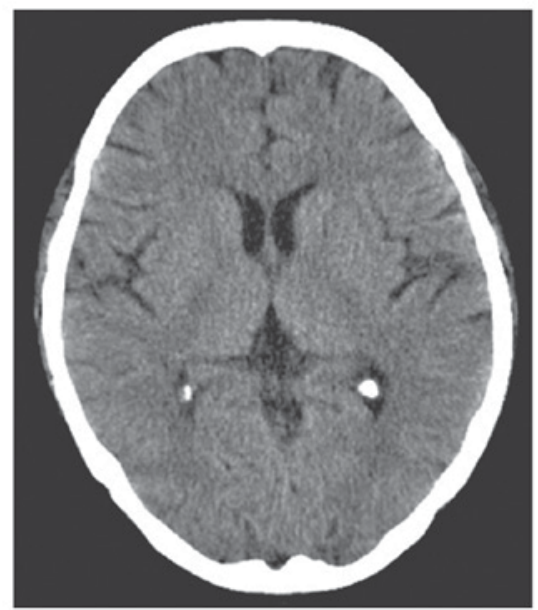

B

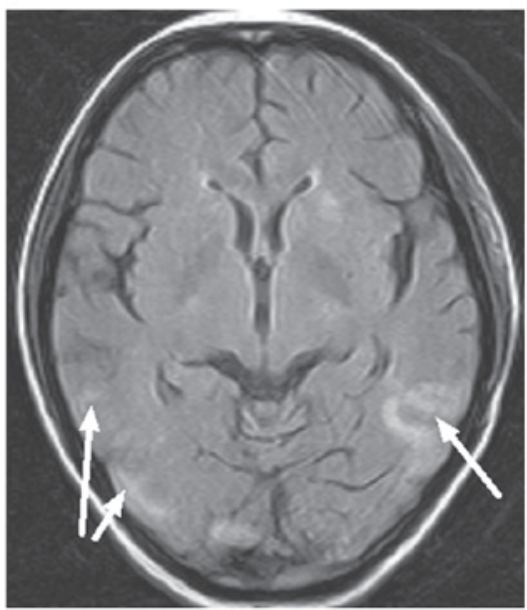

Figure 4. Two images from one female patient who was 68 years old. (A) Head CT revealed no significant low-density lesion in cerebral parenchyma. (B) Brain MRI-FLAIR demonstrated high signal intensity on bilateral temporal lobe and right occipital cortex. MRI, magnetic resonance imaging; CT, computed tomography; FLAIR, fluid-attenuated inversion recovery images.

of action may include both potential mechanisms; the specific pathophysiological mechanism of PRES remains unclear.

From the comparison of data from the two groups, average $\mathrm{Hb}$ levels in the PRES group were significantly lower than in the control group $(\mathrm{P}<0.05)$. Therefore, a lower Hb level may indicate a higher risk of PRES in patients with CRF. Anemia may be associated with ischemia and hypoxia of tissue, leading to endothelial cell injury $(31,32)$. The primary treatment of anemia is with recombinant human erytheopoietin
(rHuEPO), which may increase blood pressure and exacerbate intrinsical hypertension. It has thus been suggested that treatment with rHuEPO increases the risk of PRES in patients with CRF (33). Certain hereditary and acquired hemolytic diseases often elevate levels of circulating cell-free $\mathrm{Hb}$, which may directly contribute to liver injury by inducing oxidative tissue damage via stimulation of catalytic activity in low-density lipoprotein oxidation $(33,34)$. Consequently, the synthesis of liver-producing anticoagulation molecules such as 
anti-thrombin-III is reduced, which may result in an increase of endothelial permeability and leakage of plasma, Alb and red blood cells into the extracellular space. This may eventually induce PRES (17).

As a laboratory test providing evidence of endothelial injury (18), LDH serves an important role during the process of endothelial injury. In the current study, LDH in $76.2 \%$ (16/21) of patients with PRES rose abnormally, whereas this same rise only occurred in just $23.8 \%$ (5/21) of patients in the control group. The average LDH level in the PRES group was significantly greater than that of the control group $(\mathrm{P}<0.05)$. This strongly supports the hypothesis that for patients with $\mathrm{CRF}$, endothelial injury is one of the primary mechanisms of PRES. Meanwhile, LDH is a significant index for predicting and diagnosing of PRES early, providing a novel path for further research.

In addition, the $24 \mathrm{~h}$ urine volume in PRES group was significantly lower than that of the control group $(\mathrm{P}<0.05)$. It has been suggested that the accumulation of fluid, toxins and metabolic waste may promote the onset of PRES in patients with CRF (28). This may be due to volume-dependent hypertension caused by fluid retention and vascular endothelial injury resulting from the accumulation of toxins and metabolic waste (28). However, the specific mechanism of this action remains unclear.

In conclusion, PRES is a clinical syndrome comprised of acute neurological symptoms caused by endothelial dysfunction. This usually arises from severe abrupt arterial hypertension or fluctuations in BP, but may also be caused by direct endothelial injury resulting from the use of immunosuppressant drugs, the presence of autoimmune disorders or eclampsia. It is usually diagnosed in patents with CRF and diagnosis is difficult as CT brain scans may be normal and MRI scans are not always performed in patients with CRF. Therefore, brain MRI rather than CT scans should be performed in patients suspected of having PRES. Meanwhile, certain biochemical markers, including $\mathrm{Hb}$ and $\mathrm{Alb}$, may be associated with PRES. PRES generally has a favorable prognosis, however neurological sequelae and even fatalities may occur, particularly if the syndrome is complicated by intracranial hemorrhage or brain infarction. Thus, although knowledge regarding PRES has improved, further studies are required to elucidate its exact mechanism of action.

\section{References}

1. Campbell D and Weir MR: Defining, treating and understanding chronic kidney disease-A complex disorder. J Clin Hypertens (Greenwich) 17: 514-527, 2015.

2. Granata G, Greco A, Iannella G, Granata M, Manno A, Savastano E and Magliulo G: Posterior reversible encephalopathy syndrome-Insight into pathogenesis, clinical variants and treatment approaches. Autoimmun Rev 14: 830-836, 2015.

3. Pula JH and Eggenberger E: Posterior reversible encephalopathy syndrome. Curr Opin Ophthalmol 19: 479-484, 2008.

4. Ollivier M, Bertrand A, Clarencon F, Gerber S, Deltour S, Domont F, Trunet S, Dormont D and Leclercq D: Neuroimaging features in posterior reversible encephalopathy syndrome: A pictorial review. J Neurol Sci 373: 188-200, 2017.

5. Bartynski WS: Posterior reversible encephalopathy syndrome, part 2: Controversies surrounding pathophysiology of vasogenic edema. AJNR Am J Neuroradiol 29: 1043-1049, 2008.

6. Hinchey J, Chaves C, Appignani B, Breen J, Pao L, Wang A, Pessin MS, Lamy C, Mas JL and Caplan LR: A reversible posterior leukoencephalopathy syndrome. N Engl J Med 334: 494-500, 1996.
7. Nakabou M, Kai T, Maeshima T and Kanamasa K: Hypertensive encephalopathy in patients with chronic renal failure caused by stopping antihypertensive agents: A report of two cases. Clin Exp Nephrol 14: 256-262, 2010.

8. Zhang HL, Yang Y and Wu J: Posterior reversible encephalopathy syndrome in ANCA-associated vasculitis. Rheumatol Int 32: 1847-1848, 2012.

9. Fujieda Y, Kataoka H, Odani T, Otomo K, Kato M, Fukaya S, Oku K, Horita T, Yasuda S, Atsumi T and Koike T: Clinical features of reversible posterior leukoencephalopathy syndrome in patients with systemic lupus erythematosus. Mod Rheumatol 21: 276-281, 2011.

10. Burrus TM, Mandrekar J, Wijdicks EF and Rabinstein AA: Renal failure and posterior reversible encephalopathy syndrome in patients with thrombotic thrombocytopenic purpura. Arch Neurol 67: 831-834, 2010.

11. Liman TG, Bohner G, Heuschmann PU, Endres M and Siebert E: The clinical and radiological spectrum of posterior reversible encephalopathy syndrome: The retrospective Berlin PRES study. J Neurol 259: 155-164, 2012.

12. Kidney Disease: Improving Global Outcomes (KDIGO) CKD work group: KDIGO 2012 clinical practice guideline for the evaluation and management of chronic kidney disease. Kidney Int Suppl 3: 1-150, 2013.

13. Gao B, Liu FL and Zhao B: Association of degree and type of edema in posterior reversible encephalopathy syndrome with serum lactate dehydrogenase level: Initial experience. Eur J Radiol 81: 2844-2847, 2012.

14. Yamada A and Ueda N: Age and gender may affect posterior reversible encephalopathy syndrome in renal disease. Pediatr Nephrol 27: 277-283, 2012.

15. Fuentes AG, Komarla A and Gomez JI: Posterior reversible encephalopathy syndrome in a patient with ANCA-associated vasculitis. Rheumatol Int 32: 2529-2530, 2012.

16. Lin CY, Chen HC, Hwang WL and Teng CL: Immune thrombocytopenic pupura-induced reversible posterior leukoencephalopathy successfully treated by rituximab. Ann Hematol 90: 731-732, 2011.

17. Piccin A, Dossi RC, Cassibba V, Stupnner S, Bonatti G and Cortelazzo S: Anti-thrombin-III reduction and posterior reversible encephalopathy syndrome (PRES) in acute lymphoblastic leukaemia (ALL). New insight into PRES pathophysiology. Ann Hematol 91: 1153-1155, 2012.

18. Hadj JO, Braven RD, Tillier C, Schrijver HM, Verheul HM and VAN DER Vliet HJ: Reversible posterior leukoencephalopathy syndrome during sunitinib therapy for metastatic renal cell carcinoma. Oncol Lett 3: 1293-1296, 2012.

19. Nasu K, Fujisawa M, Kato H and Nangaku M: Three cases of posterior reversible encephalopathy syndrome with chronic kidney disease triggered by infection. Nephrology (Carlton) 22: 322-325, 2017.

20. Canney M, Kelly D and Clarkson M: Posterior reversible encephalopathy syndrome in end-stage kidney disease: Not strictly posterior or reversible. Am J Nephrol 41: 177-182, 2015.

21. Vargas M, Servillo G and Striano P: Serum lactate dehydrogenase as early marker of posterior reversible encephalopathy syndrome: Keep your eyes open. Anaesth Intensive Care 40: 570-571, 2012.

22. Pawlak K, Naumnik B, Brzósko S, Pawlak D and Myśliwiec M: Oxidative stress-a link between endothelial injury, coagulation activation, and atherosclerosis in haemodialysis patients. Am J Nephrol 24: 154-161, 2004.

23. Stenvinkel P: Endothelial dysfunction and inflammation-is there a link? Nephrol Dial Transplant 16: 1968-1971, 2001.

24. Tepperman E, Ramzy D, Prodger J, Sheshgiri R, Badiwala M, Ross H and Raoa V: Surgical biology for the clinician: Vascular effects of immunosuppression. Can J Surg 53: 57-63, 2010.

25. Yoon HE and Yang CW: Established and newly proposed mechanisms of chronic cyclosporine nephropathy. Korean J Intern Med 24: 81-92, 2009.

26. Huynh TN, Chacko BK, Teng X, Brott BC, Allon M, Kelpke SS, Thompson JA, Patel RP and Anayiotos AS: Effects of venous needle turbulence during ex vivo hemodialysis on endothelial morphology and nitric oxide formation. J Biomech 40: 2158-2166, 2007.

27. Lilien MR, Koomans HA and Schröder CH: Hemodialysis acutely impairs endothelial function in children. Pediatr Nephrol 20: 200-204, 2005.

28. Li R, Mitchell P, Dowling R and Yan B: Is hypertension predictive of clinical recurrence in posterior reversible encephalopathy syndrome? J Clin Neurosci 20: 248-252, 2013. 
29. Siddall EC and Radhakrishnan J: The pathophysiology of edema formation in the nephrotic syndrome. Kidney Int 82: 635-642, 2012.

30. Mackow ER, Gorbunova EE and Gavrilovskaya IN: Endothelia cell dysfunction in viral hemorrhage and edema. Front Microbiol 5: 733, 2015.

31. Asikainen TM, Ahmad A, Schneider BK, Ho WB, Arend M, Brenner M, Günzler V and White CW: Stimulation of HIF-1alpha, HIF-2alpha and VEGF by prolyl 4-hydroxylase inhibition in human lung endothelial and epithelial cells. Free Radic Biol Med 38: 1002-1013, 2005.

32. Delanty N, Vaughan C, Frucht S and Stubgen P: Erythropoietin-associated hypertensive posterior leukoencephalopathy. Neurology 49: 686-689, 1997.
33. Miller YI, Altamentova SM and Shaklai N: Oxidation of low-density lipoprotein by hemoglobin stems from a heme-initiated globin radical: Antioxidant role of haptoglobin. Biochemistry 36: 12189-12198, 1997.

34. Belcher JD, Beckman JD, Balla G, Balla J and Vercellotti G: Heme degradation and vascular injury. Antioxid Redox Signal 12: $233-248,2010$ 